This article was downloaded by:[University of Waterloo]

On: 16 May 2007

[University of Waterloo]

Access Details: [subscription number 769429802]

Publisher: Taylor \& Francis

Informa Ltd Registered in England and Wales Registered Number: 1072954

Registered office: Mortimer House, 37-41 Mortimer Street, London W1T 3J H, UK

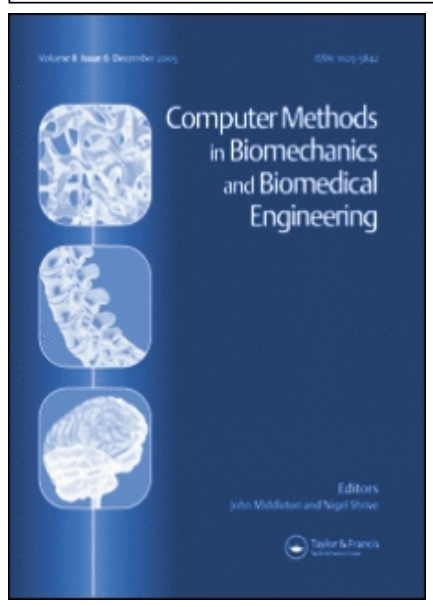

\title{
Computer Methods in Biomechanics and Biomedical Engineering
}

Publication details, including instructions for authors and subscription information: http://www.informaworld.com/smpp/title $\sim$ content=t713455284

Estimation of cellular fabric in embryonic epithelia

To cite this Article: Iles, Peter J. W., Brodland, G. Wayne, Clausi, David A. and

Puddister, Shannon M. , 'Estimation of cellular fabric in embryonic epithelia',

Computer Methods in Biomechanics and Biomedical Engineering, 10:1, 75 - 84

To link to this article: DOI: $10.1080 / 10255840601066848$

URL: http://dx.doi.org/10.1080/10255840601066848

\section{PLEASE SCROLL DOWN FOR ARTICLE}

Full terms and conditions of use: $h \mathrm{htp}: / / w w w$.informaworld.com/terms-and-conditions-of-access.pdf

This article maybe used for research, teaching and private study purposes. Any substantial or systematic reproduction, re-distribution, re-selling, loan or sub-licensing, systematic supply or distribution in any form to anyone is expressly forbidden.

The publisher does not give any warranty express or implied or make any representation that the contents will be complete or accurate or up to date. The accuracy of any instructions, formulae and drug doses should be independently verified with primary sources. The publisher shall not be liable for any loss, actions, claims, proceedings, demand or costs or damages whatsoever or howsoever caused arising directly or indirectly in connection with or arising out of the use of this material.

(c) Taylor and Francis 2007 


\title{
Estimation of cellular fabric in embryonic epithelia
}

\author{
PETER J. W. ILES $\dagger$, G. WAYNE BRODLAND $\ddagger *$, DAVID A. CLAUSI $\dagger$ and SHANNON M. PUDDISTER $\dagger$ \\ $\dagger$ Department of Systems Design Engineering, University of Waterloo, Waterloo, Ont., Canada N2L 3G1 \\ \$Department of Civil and Environmental Engineering, University of Waterloo, Waterloo, Ont., Canada N2L 3G1
}

(Received 1 February 2006; in final form 18 September 2006)

\begin{abstract}
Recent computational and analytical studies have shown that cellular fabric-as embodied by average cell size, aspect ratio and orientation-is a key indicator of the stresses acting in an embryonic epithelium. Cellular fabric in real embryonic tissues could not previously be measured automatically because the cell boundaries tend to be poorly defined, significant lighting and cell pigmentation differences occur and tissues contain a variety of cell geometries. To overcome these difficulties, four algorithms were developed: least squares ellipse fitting (LSEF), area moments (AM), correlation and axes search (CAS) and Gabor filters (GF). The AM method was found to be the most reliable of these methods, giving typical cell size, aspect ratio and orientation errors of $18 \%, 0.10$ and $7.4^{\circ}$, respectively, when evaluated against manually segmented images. The power of the AM algorithm to provide new insights into the mechanics of morphogenesis is demonstrated through a brief investigation of gastrulation, where fabric data suggest that key gastrulation movements are driven by epidermal tensions circumferential to the blastopore.
\end{abstract}

Keywords: Cellular fabric; Tissue mechanics; Image processing; Embryogenesis

\section{Introduction}

During early embryo development, sheets of cells called epithelia must undergo precise, self-driven morphogenetic movements if a normal embryo is to be formed (Jacobson 1962, Jacobson and Gordon 1976, Keller 1978, Trinkaus 1984, Gilbert 2003). Irregularities in these movements can give rise to serious birth defects such as spina bifida, cardiac septum defects and cleft lip and palate. In order to understand the mechanics of normal development and the causes of these often severely debilitating defects, it is necessary to quantify the mechanical properties of the embryonic tissues involved and the stresses and strains present in them during morphogenetic movements.

Recent computational simulations (Chen and Brodland 2000, Brodland and Veldhuis 2002) and theoretical analyses (Brodland et al. 2000, Brodland and Wiebe 2004, Brodland et al. 2006) have shed light on the interrelationships between tissue mechanics and cellular fabric. These studies have shown that the mechanical properties of tissues are directly related to their cellular fabric, as characterized by average cell size, aspect ratio and orientation. They have also shown that the cellular fabric is dependent on the recent strain history of the tissue, as well as other factors such as mitosis rate.
Consequently, if the cellular fabric of a tissue can be accurately tracked over a period of time, important inferences can be made regarding its stress state, strain history and mechanical properties. Even subtle fabric anisotropies that are not being detectable by visual inspection may have important mechanical consequences within the embryo.

These computational and analytical studies have also shown that cellular fabric can be embodied in an elliptical 'composite cell' (figure 1) whose area, aspect ratio and orientation are an average of those of the cells in the region of interest. The major axis of the composite cell is assumed to be oriented counterclockwise from the $x$-axis by angle $-90^{\circ}<\alpha<90^{\circ}$ and to have major and minor axes of lengths $L_{\text {major }}$ and $L_{\text {minor }}$, respectively. We define the aspect ratio $\kappa \geq 1$ of the composite cell to be

$$
\kappa=\frac{L_{\text {major }}}{L_{\text {minor }}}
$$

and its area $A$ is given by

$$
A=\left(\frac{\pi}{4}\right) L_{\text {major }} L_{\text {minor }} .
$$

In practice, we define the cellular fabric in terms of $\alpha, \kappa$ and $A$, rather than the ellipse dimensions.

*Corresponding author. Email: brodland@uwaterloo.ca 


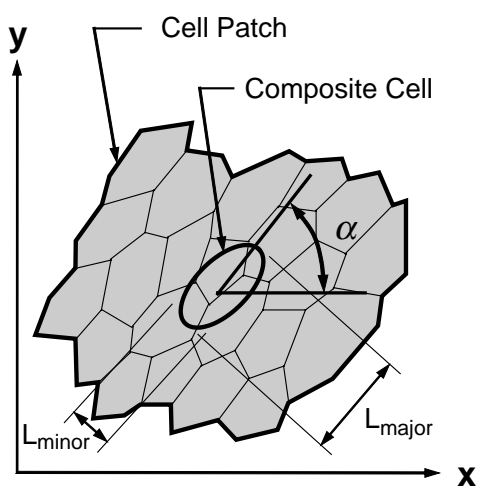

Figure 1. The composite cell associated with a cell patch is defined by its orientation $\alpha$ and the lengths of its major and minor axes, $L_{\text {major }}$ and $L_{\text {minor }}$, respectively.

Recent constitutive equation derivations for embryonic tissues (Brodland et al., 2006) have shown that the mesocharacteristics of fundamental importance to cell mechanics are the statistical distributions of cell edge lengths and orientations. Thus, it is not the overall dimensions of a cell or its actual outline that are important, but rather the lengthweighted angular distribution of its edges. From the standpoint of characterizing epithelia, this is an important practical finding because it shows that complete closed cell outlines are not required. Instead, it is sufficient that the visible boundaries represent statistically the lengths and angular distributions of all of the boundaries in the tissue. This requirement is substantially less stringent than that of other methods (Ranefall et al. 1997, Wählby et al. 2002), which typically require that cell contours be closed.

These derivations have shown that cell edges are distributed homologously with those of the composite cell (Brodland and Wiebe 2004). Consequently, if one could determine the length-weighted angular distribution of the cell edges in a tissue, one could directly obtain a unique composite cell that would be representative of that tissue. Fortunately, an elliptically-distributed edge distribution is amenable to detection by frequency-domain image processing methods.

Automating the extraction of cell fabric data is important because during a typical study of embryo development, hundreds of images may be collected from a single embryo. Since more than an hour is required to digitize manually the edges in a typical image, this method is impractical for detailed investigations of cell shapes. In addition, automation eliminates human bias and error, especially when subtle anisotropies and regional variations may be of interest.

Although image processing methods have been used to analyze cells for a number of years, existing methods work only in cases where cells do not touch each other (Mukherjee et al. 2004), have very high contrast (YingLun et al. 1996) or have well-defined, closed boundaries (Ying-Lun et al. 1996, Lin et al. 1998, Mukherjee et al. 2004). In the study of embryonic tissues (figure 2), these criteria are not satisfied because the cell boundaries tend to be poorly defined and significant lighting and cell pigmentation differences can occur.
One might consider using texture analysis methods (Matsuyama et al. 1983, Kim and Park 1992, Starovoitov et al. 1998), but they assume regular repetition of a fixed "texture element" within the image, and that is not the case in images of embryonic tissues. Watershed methods have also been successfully applied to a number of cell analysis problems (Ranefall et al. 1997, Wählby et al. 2002), but they require that the cell boundaries be well defined, a criterion seldom met in images of embryonic tissues.

The purpose of this paper is to develop an algorithm that can estimate the composite cell properties $\alpha, \kappa$ and $A$ to within one standard error of the corresponding best estimates $\hat{\alpha}, \hat{\kappa}$ and $\hat{A}$ obtained by statistical analysis of manually traced cells. Four different algorithms that use frequency domain concepts to estimate these properties are proposed. These methods are least squares ellipse fitting (LSEF), area moments (AM), correlation and axes search (CAS), and Gabor filters (GF). Block diagrams for the first three methods are shown in figure 3.

The methods were evaluated by applying them to a set of 16 images (figure 2) that illustrate the range of images typically obtained from embryos of the axolotl, an amphibian commonly used to investigate morphogenetic movements. The cells of axolotl embryos, unlike those of most other vertebrates, have a noticeable degree of natural colouration. In the absence of natural colouration, cell boundaries can be elucidated by staining.

Images were collected from live embryos using a robotic microscope system (Veldhuis et al. 2005) and from excised pieces of epithelium that had been stretched in a novel tensile test instrument (Wiebe and Brodland, in press). Figure 2 demonstrates that even within a single image, substantial variability can occur in illumination (figure 2(a)), cell pigmentation (figure 2(c)), cell boundary visibility (figure $2(\mathrm{f})$ ) and cell orientation and shape (figure 2(k)), posing significant challenges that the algorithms must overcome.

Although the AM algorithm was found to be the most reliable of the algorithms tested, the LSEF and CAS algorithms also demonstrated acceptable performance in many cases. The AM algorithm was used to measure the fabric of epithelium adjacent to the blastopore of a stage 12.5 axolotl embryo during a crucial developmental process called gastrulation. The stresses acting in the epithelium were calculated based on the fabric data, and they show that substantial circumferential tensions exist in the epithelium that surrounds the blastopore.

\section{Methods}

\subsection{Frequency domain analysis}

Figure 4(a) shows an image of a synthetic cell patch in term of $x-y$ axes and its corresponding magnitude response image (figure 4(b)) formed by taking the two-dimensional fast Fourier transform (FFT) of the square source image. The magnitude response image $|F|$ is presented in terms of 


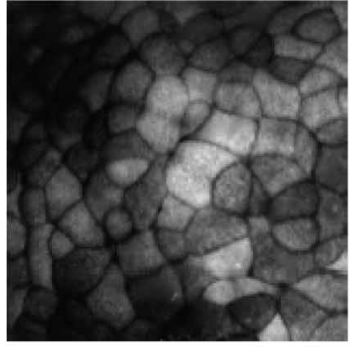

(a) $480 \times 480$

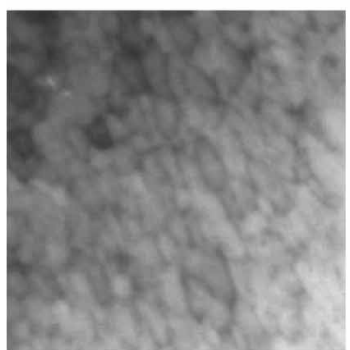

(e) $316 \times 316$

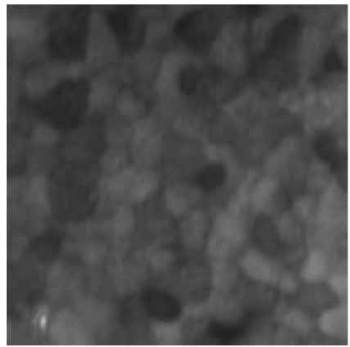

(i) $237 \times 237$

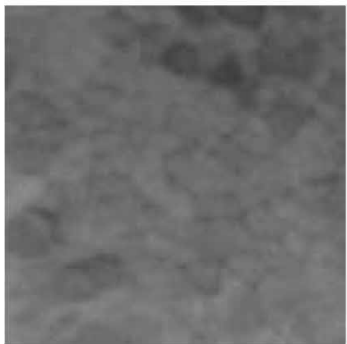

(m) $162 \times 162$

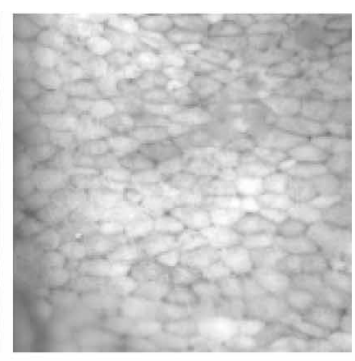

(b) $672 \times 672$

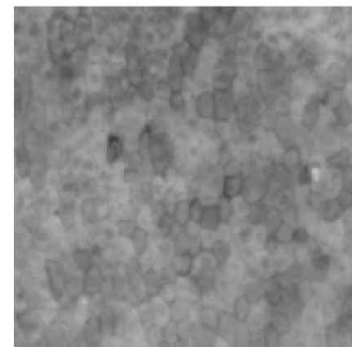

(f) $577 \times 577$

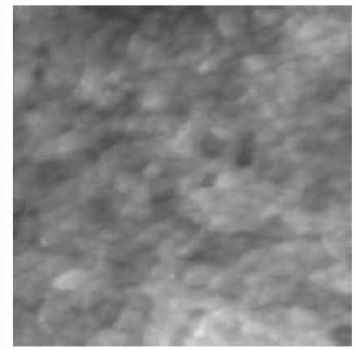

(j) $302 \times 302$

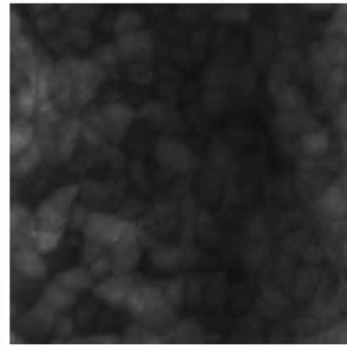

(n) $290 \times 290$

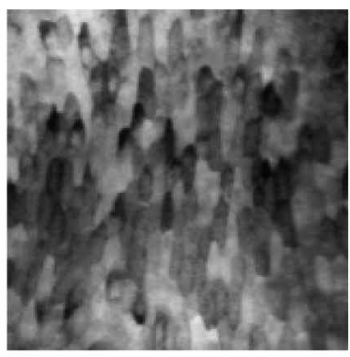

(c) $349 \times 349$

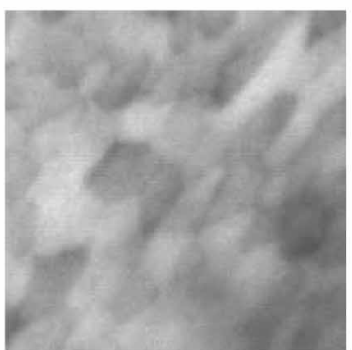

(g) $214 \times 214$

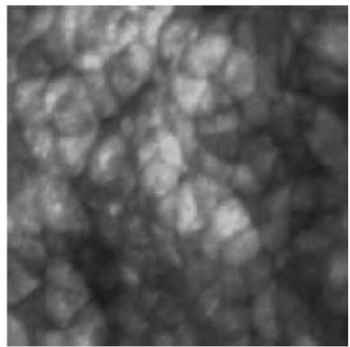

(k) $241 \times 241$

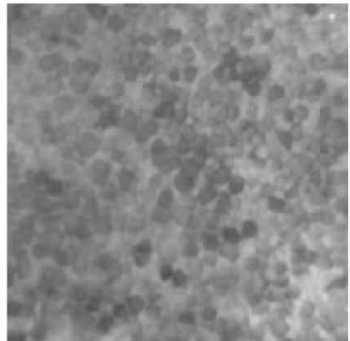

(o) $367 \times 367$

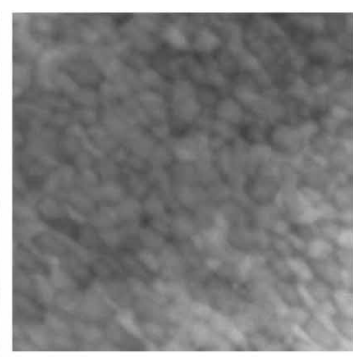

(d) $385 \times 385$

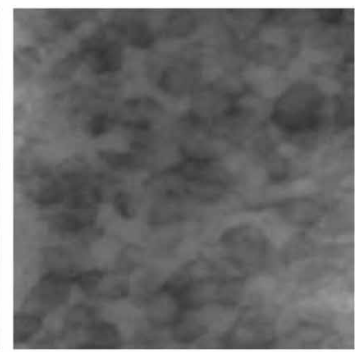

(h) $232 \times 232$

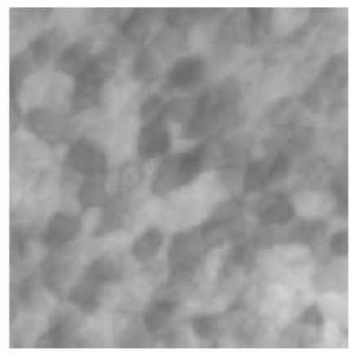

(I) $233 \times 233$

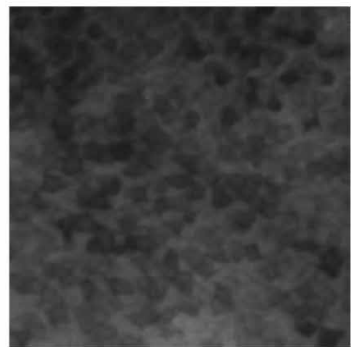

(p) $320 \times 320$

Figure 2. Representative images of embryonic epithelia. Numbers indicate the size of pixels in each image.

$u-v$ axes in pixel units of cycles per image (c.p.i). To generate the patch, a Voronoi tessellation was constructed from forming points whose minimum spacing had been limited so that cells of approximately uniform size resulted (Chen and Brodland 2000). The patch was then elongated in the $x$-direction by a factor of 1.8 so as to produce an anisotropic fabric. The statistical distribution of the cell boundaries in the patch will be the same as the infinitesimal perimeter segments of the ellipse corresponding to the composite cell (Brodland and Wiebe 2004).

The ellipse contained in the magnitude response image $|F|$ is normal to the composite cell and its major and minor axes are the multiplicative inverses of the composite cell axes. This duality arises because the strong high-frequency response in the vertical direction (along the $v$ axis) in the $|F|$ image arises from the high density of cell edges encountered in the vertical direction, while the weaker low-frequency response in the horizontal direction (along the $u$-axis) is produced by the less frequent cell edges in the horizontal direction. Because of this duality, the composite cell axis lengths and orientations could be obtained if one could fit an ellipse to the elliptic shape in the magnitude response image. 

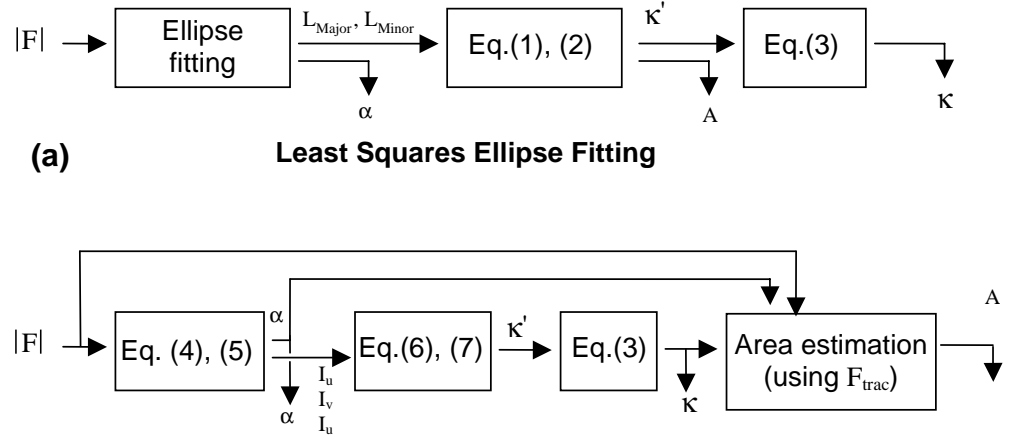

(b)

Area Moments (AM)

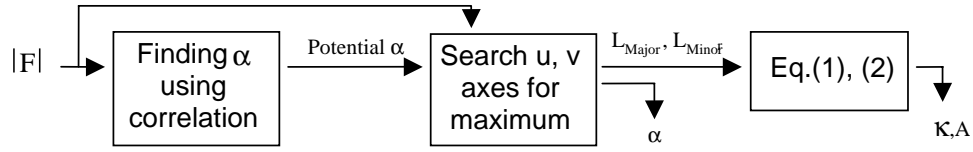

(c)

Correlation and Axes

Figure 3. Block diagrams for the LSEF, AM and CAS algorithms.

A benefit of frequency-domain methods is that local variations over the spatial region of interest are averaged out by the integrals associated with the temporalfrequency transformation. The strongest responses in the $|F|$ image reveal the "average" cell orientation and axis lengths, which is the goal of the analysis. This effect makes these methods robust to spatial variations in cell shape, orientation and edge visibility.

\subsection{Image pre-processing}

Four steps were used to pre-process images before one of the four ellipse extraction techniques was applied. These steps were:

(1) Calculate the gradient magnitude image (figure 5(a)). Horizontal and vertical Sobel operators (Castleman 1996) were applied to the source image to highlight any visible cell boundaries (which contain the frequency content that is interpreted by the FFT) and reduce the effect of cell-wise differences in pigmentation. The filtered results were combined to form a gradient magnitude image.

(2) Transform image to frequency domain. An FFT was then taken of the resulting gradient image to create the magnitude response image $|F|$. To avoid angular bias, the square $|F|$ image was then cropped along its maximal contained circle.

(3) Intensity-threshold $|F|$. Next, the $|F|$ image was normalised to $[0,1]$ and thresholded by setting to zero components below $F_{\text {thresh }}=0.30$, a threshold that was found to work well with all of the ellipse extraction methods. The purpose of this step is to remove low-energy noise.
(4) Frequency-threshold $|F|$ (figure 5(b)). Frequency components at or below four cycles per image were set to zero since these components often contain substantial energy but are associated with spatial patterns too large to be individual cells. This step also removed substantial artifacts that could otherwise be produced by low-frequency illumination variations.

\subsection{Method 1: least squares ellipse fitting (LSEF)}

Least squares methods provide a standard approach for curve and surface fitting (Fitzgibbon et al. 1999). To this end, the data $|F|$ in the magnitude response image were converted into a list of data points by creating at each pixel location, one data point for each 0.1 of pixel intensity. Thus, a pixel with an intensity of 0.4 would be converted into four data points. Fitting of an ellipse to the points is then done using the approach of Fitzgibbon et al. (1999) because it is robust and computationally inexpensive. Once an ellipse is fit to the data, $\alpha, L_{\text {major }}$ and $L_{\text {minor }}$ can be calculated directly and $\kappa$ and $A$ can be calculated using equations (1) and (2), respectively.

The values of $\kappa$ obtained using this algorithm were found to be biased high compared to values obtained by manually tracing the cells in the image (figure 6). This overestimation occurs because the energy corresponding to $L_{\text {minor }}$ in the $|F|$ image was larger than the energy corresponding to $L_{\text {major }}$. As a result, the fitted ellipse was consistently "stretched" somewhat in the $L_{\text {minor }}$ direction. A linear function,

$$
\kappa=\kappa^{\prime}-m\left(\kappa^{\prime}-1\right)
$$

was found to correct $\kappa^{\prime}$, the biased estimate, and produce an unbiased result, $\kappa$. An $m$ value of 0.29 was found to minimize 
(a)

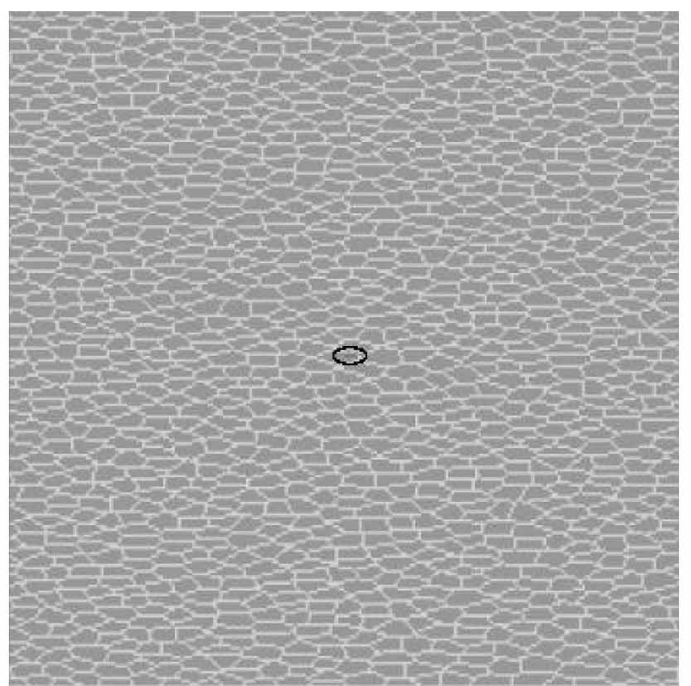

$v$

(b)

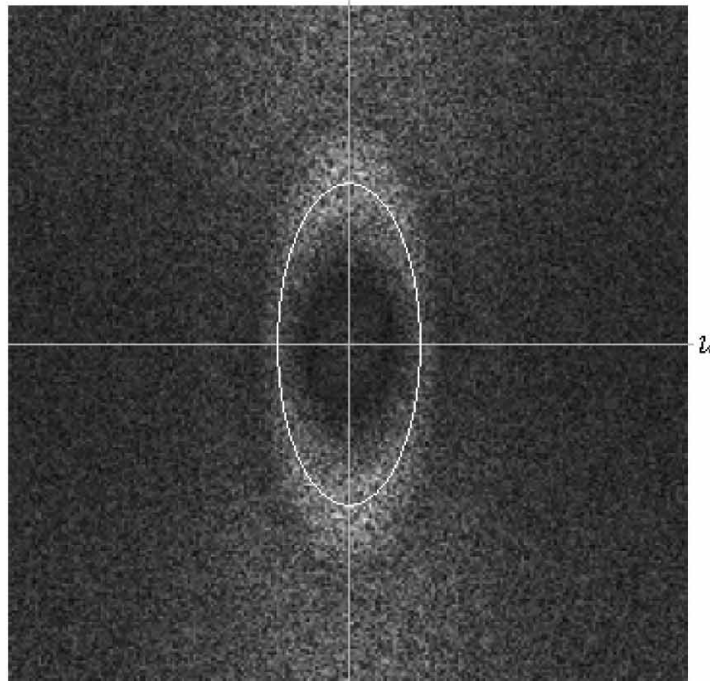

Figure 4. An image of a cellular tissue and its corresponding magnitude response image $|\mathrm{F}|$. (a) A synthetic $350 \times 350$ pixel cellular image with $\alpha=0^{\circ}, \kappa=1.8$ and $A=24$ pixels. The composite cell is drawn in black. (b) Corresponding magnitude response $(F)$ image in pixel units of cycles per image. A square root point operator has been applied to enhance visibility. The transform of the composite cell is shown as a white ellipse.

the least-squared error between the LSEF estimates and the values found by manual segmentation of the images shown in figure 2. This bias was found to be inherent to the algorithm rather than to the images, and hence a constant $m$ value could be chosen.

\subsection{Method 2: area moments (AM)}

The data in the $|F|$ image can be thought of as a twodimensional object of varying density (as defined by the pixel intensities) centred at the origin. The idea of using AM of the $|F|$ image to extract texture orientation was suggested by Bigün et al. (1991). Their method solves a matrix eigenvalue problem in the spatial domain. Instead, we follow Brodland and Veldhuis (2003), and calculate the $\mathrm{AM} I_{v}$ and $I_{u}$ about the $u$ - and $v$-axes, respectively, and the product of inertia $I_{u v}$ of the $|F|$ image using the (a)

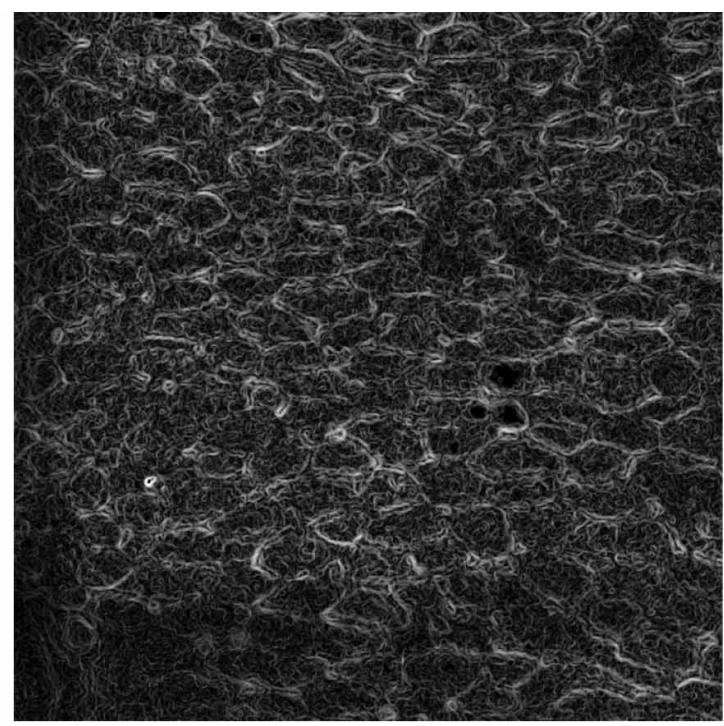

(b)

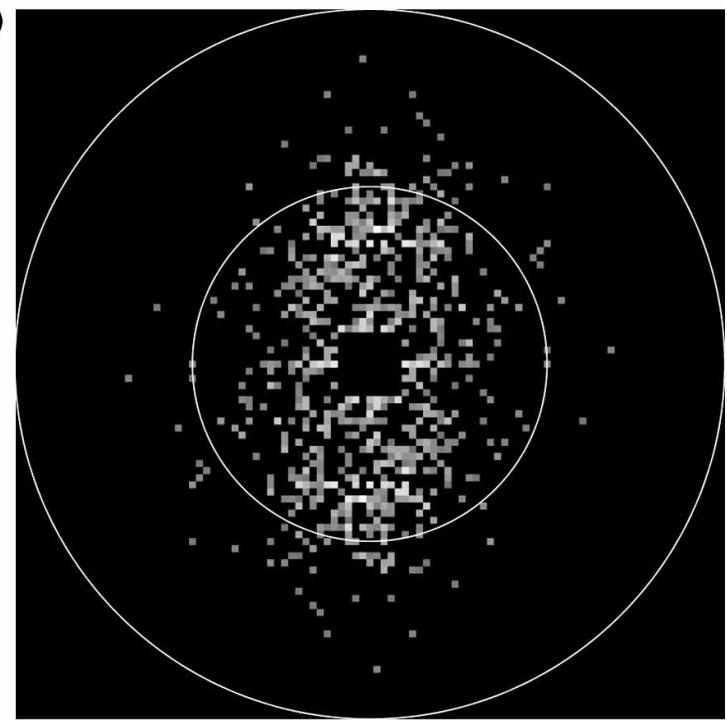

Figure 5. Selected pre-processing steps for the test image shown in figure $2(b)$ : (a) the gradient magnitude image and (b) the intensity- and frequency-thresholded $F$ image. Concentric circles show 25 and 50 c.p.i.

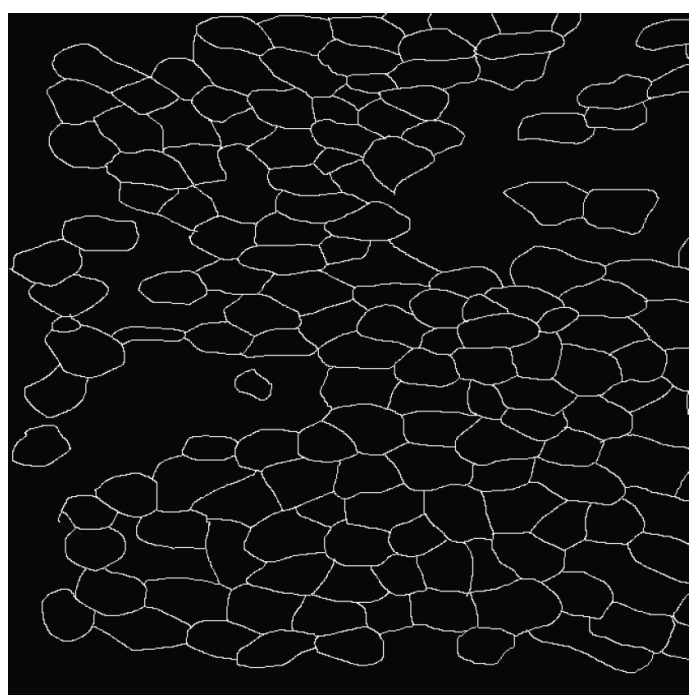

Figure 6. Manually segmented image corresponding to figure 2(b). 
formulae (Hibbeler 2005)

$$
\begin{aligned}
& I_{u}=\sum_{v=1}^{\text {rows }} \sum_{u=1}^{\text {cols }}|F(u, v)| \cdot v^{2}, \\
& I_{v}=\sum_{v=1}^{\text {rows }} \sum_{u=1}^{\text {cols }}|F(u, v)| \cdot u^{2}, \\
& I_{u v}=\sum_{v=1}^{\text {rows }} \sum_{u=1}^{\text {cols }}|F(u, v)| \cdot u v
\end{aligned}
$$

Next, $\alpha$ is computed using

$$
\alpha=\arctan \left(\frac{2 I_{u v}}{I_{u}-I_{v}}\right),
$$

provided that $I_{u} \neq I_{v}$. In the case that $I_{u}=I_{v}$, we set

$$
\alpha= \begin{cases}\frac{\pi}{4}, & I_{u v}>0 \\ 0, & I_{u v}=0 \\ -\frac{\pi}{4}, & I_{u v}<0 .\end{cases}
$$

Mohr's circle (Hibbeler 2005), can then be used to extract the principal moments

$$
I_{1}=\frac{I_{u}+I_{v}}{2}+\sqrt{\left(\frac{I_{u}-I_{v}}{2}\right)^{2}+I_{u v}^{2}}
$$

and

$$
I_{2}=I_{u}+I_{v}-I_{1}
$$

and the aspect ratio, $\kappa$, calculated using

$$
\kappa=\frac{\max \left(\sqrt{I_{1}}, \sqrt{I_{2}}\right)}{\min \left(\sqrt{I_{1}}, \sqrt{I_{2}}\right)}
$$

The AM method, like the LSEF approach produces $\kappa$ values that are biased high, and that can be corrected optimally using equation (3) when $m$ is set to 0.38 .

The composite cell area, $A$, was estimated by finding, from the family of ellipses constrained to the estimated $\alpha$ and $\kappa$, the one that contained a fraction $F_{\text {frac }}$ of the energy in the $|F|$ image. The fraction that minimized the error for the test images was found to be $F_{\text {frac }}=0.28$.

The error in $A$ was found to be sensitive to the number of cells in the image. When an image of a particular region was expanded to encompass more cells, there was more low frequency noise present in $|F|$. This caused the ellipse containing the specified fraction of the total energy in the frequency domain to become too small and the corresponding composite cell to become too large. A correlation was observed between this effect and the average c.p.i. $(\bar{c})$ of the $|F|$ image, thus providing a means to overcome this difficulty. It was found experimentally that when the low frequency threshold in Step 3 of the preprocessing was set to

$$
F_{\text {low }}^{\prime}=\frac{\bar{c}}{3}
$$

an appropriate amount of the low frequency energy was removed that the calculated $A$ values were largely insensitive to the number of cells in the image, provided that images had an $L_{\text {minor }}$ in the range of 10-22 c.p.i., a range of values that is wide enough that it can be satisfied by a user during manual selection of an analysis window.

\subsection{Method 3: correlation and axes search (CAS)}

The third frequency domain approach estimated $\alpha$ by identifying the line of symmetry in the $|F|$ image (Bigün et al. 1991). To find the angle of this line, we use the correlation coefficient, $-1<\rho<1$, defined for two data vectors, $a$ and $b$ as

$$
\rho=\frac{E\left\{\left(a-\mu_{a}\right)\left(b-\mu_{b}\right)\right]}{\sqrt{\operatorname{var}(a) \operatorname{var}(b)}}
$$

where $E[$.$] is the expectation operator, \operatorname{var}($.$) is the sample$ variance operator, and $\mu_{a}$ and $\mu_{b}$ are the sample means for $a$ and $b$, respectively.

In this approach, a series of new images is created by incrementally rotating $|F|$ through $1^{\circ}<\mu<90^{\circ}$. After each rotation, $\rho$ is calculated between mirrored pixels on the left and right sides of the image using equation (9). This is done by placing mirrored pixels from the left and right halves of the image into corresponding locations in vectors $a$ and $b$, respectively. When the $|F|$ ellipse is aligned (either horizontally or vertically) with the $u$ and $v$ axes, $\rho$ is maximized (figure 7). The axis lengths are then found by searching for maxima along two rays in the $|F|$ image, one parallel to and one perpendicular to the line of symmetry. From these, $L_{\text {major }}$ and $L_{\text {minor }}$ are calculated, and $\kappa$ and $A$ are found using equations (1) and (2), respectively. The orientation, $\alpha$, is then calculated as the angle corresponding to $L_{\text {major }}$ in the frequency domain.

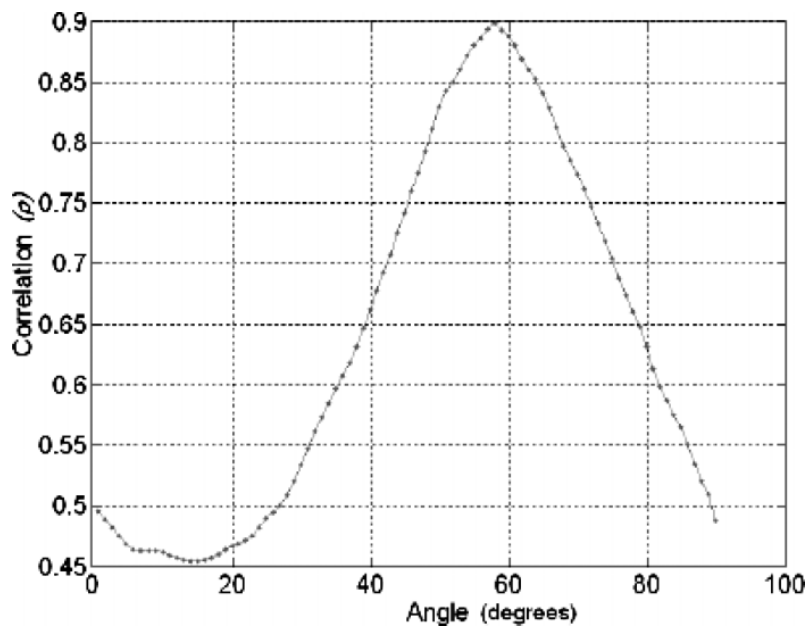

Figure 7. Estimating $\alpha$ using correlation. In this example the correlation $\rho$ reaches a well-defined maximum when the $|F|$ image ellipse is aligned with either the horizontal or vertical axis. 
Table 1. Distance from $\hat{\alpha}$ for each method in degrees $\left(\varepsilon_{\alpha}=\alpha-\hat{\alpha}\right)$ and number of standard deviations $\left(\zeta_{\alpha}=\varepsilon_{\alpha} / \sigma_{\hat{\alpha}}\right) \hat{\text { for test images. }}$

\begin{tabular}{|c|c|c|c|c|}
\hline Image & $\hat{\alpha}, \sigma_{\alpha}$ & $\begin{array}{c}\text { LSEF } \\
\varepsilon_{\alpha}\left(\zeta_{\alpha}\right)\end{array}$ & $\begin{array}{c}\mathrm{AM} \\
\varepsilon_{\alpha}\left(\zeta_{\alpha}\right)\end{array}$ & $\begin{array}{c}\text { CAS } \\
\varepsilon_{\alpha}\left(\zeta_{\alpha}\right)\end{array}$ \\
\hline A & $36.8,49.2$ & $+29.2(+0.59)$ & $+21.4(+0.43)$ & $+15.2(+\mathbf{0 . 3 1})$ \\
\hline B & $-6.9,16.1$ & $-3.7(-0.23)$ & $+1.7(+\mathbf{0 . 1 1})$ & $+2.9(+0.18)$ \\
\hline $\mathrm{C}$ & $85.9,17.6$ & $-1: 1(-0.06)$ & $+0.1(+\mathbf{0 . 0 1})$ & $+0.1(+\mathbf{0 . 0 1})$ \\
\hline $\mathrm{D}$ & $-39.0,33.1$ & $-3: 6(-\mathbf{0 . 1 1})$ & $-3.7(-0.11)$ & $-5.0(-0.15)$ \\
\hline $\mathrm{E}$ & $-60.6,27.6$ & $+2: 6(+0.09)$ & $+1.6(+\mathbf{0 . 0 6})$ & $-2.4(-0.09)$ \\
\hline $\mathrm{F}$ & $87.8,21.6$ & $-7.0(-0.32)$ & $-7.5(-0.35)$ & $-0.8(-\mathbf{0 . 0 4})$ \\
\hline G & $52.4,17.3$ & $-1: 9(-\mathbf{0 . 1 1})$ & $-2.9(-0.17)$ & $-3.4(-0.20)$ \\
\hline $\mathrm{H}$ & $11.3,34.3$ & $+0.8(+\mathbf{0 . 0 2})$ & $+1.1(+0.03)$ & $+2.7(+0.08)$ \\
\hline I & $75.5,40.2$ & $-2.8(-0.07)$ & $-1.3(-\mathbf{0 . 0 3})$ & $+5.5(+0.14)$ \\
\hline $\mathrm{J}$ & $22.7,25.7$ & $-1.4(-\mathbf{0 . 0 5})$ & $-1.7(-0.07)$ & $-2.7(-0.11)$ \\
\hline $\mathrm{K}$ & $-88.9,38.8$ & $-23.1(-0.60)$ & $-14.9(-\mathbf{0 . 3 8})$ & $+21.9(+0.56)$ \\
\hline $\mathrm{L}$ & $53.3,35.2$ & $+3.1(+0.09)$ & $+0.9(+\mathbf{0 . 0 3})$ & $+2.7(+0.08)$ \\
\hline $\mathrm{M}$ & $-24.8,39.5$ & $+18.9(+0.48)$ & $+17.4(+0.44)$ & $+15.8(+\mathbf{0 . 4 0})$ \\
\hline $\mathrm{N}$ & $82.3,51.2$ & $-22.6(-\mathbf{0 . 4 4})$ & $-27.0(-0.53)$ & $-49.3(-0.96)$ \\
\hline $\mathrm{O}$ & $75.2,44.9$ & $-2.5(+\mathbf{0 . 0 6})$ & $-3.2(-0.07)$ & $+9.8(+0.22)$ \\
\hline \multirow[t]{2}{*}{$\mathrm{P}$} & $-2.3,46.1$ & $+10.5(+0.23)$ & $+12.5(+0.27)$ & $+8.3(+\mathbf{0 . 1 9})$ \\
\hline & Average absolute & $8.4(0.22)$ & $7.4(\mathbf{0 . 1 9})$ & $9.3(0.23)$ \\
\hline
\end{tabular}

Best results for each image are shown in bold.

\subsection{Method 4: Gabor filters (GF)}

GF are a classic approach for extracting textural information from images (Bovik et al. 1990, Clausi and Jernigan 2000). Inasmuch as the cellular features of interest are 'texture-like', one might expect them to be able to extract the required cell parameters (Puddister 2003). Initial testing showed that they work well if the cell arrangements are highly regular but not if they contain the irregularities common to images of real tissues. As a result, GF were deemed unsuitable for the applications of interest here and are not discussed further.

\section{Evaluation of the algorithms}

To provide data against which the image analysis algorithms could be evaluated, a set of 16 test images (figure 3) was hand-segmented (figure 6) and the statistical properties of the cells in each image calculated. Only cells that were completely inside the image were segmented and, on occasion, portions of the cell-cell boundaries had to be estimated. The average moments and products of inertia calculated for each cell were used in equations (4) -(7) to extract best estimates $\hat{\alpha}$ and $\hat{\kappa}$ of cell orientation and aspect ratio, respectively, and $\hat{A}$ was set equal to the average area of the manually segmented cells. It is understood that manual segmentation may introduce a small amount of error, but it is the best method available for estimating the true statistical properties of the cells.

Tables 1-3 summarize how each of the three methods performed on the test images. For each image, the best estimate of the appropriate parameter $\hat{\alpha}$ and $\hat{\kappa}$ or $\hat{A}$ is shown in the leftmost column, as is its standard error. These means and standard errors were estimated by statistical analysis of the manually-segmented cells. The discrepancies between these best estimates and the values calculated by the algorithms are reported as signed values and as a factor normalized against the image-specific standard

Table 2. Distance for each method in dimensionless units $\left(\varepsilon_{\kappa}=\kappa-\hat{\kappa}\right)$ and number of standard deviations $\left(\zeta_{\alpha}=\varepsilon_{\kappa} / \sigma_{\hat{\kappa}}\right)$.

\begin{tabular}{|c|c|c|c|c|}
\hline Image & $\hat{\kappa}, \sigma_{\hat{\kappa}}$ & $\begin{array}{l}\text { LSEF } \\
\varepsilon_{\kappa}\left(\zeta_{\kappa}\right)\end{array}$ & $\begin{array}{c}\mathrm{AM} \\
\varepsilon_{\kappa}\left(\zeta_{\kappa}\right)\end{array}$ & $\begin{array}{c}\text { CAS } \\
\varepsilon_{\kappa}\left(\zeta_{\kappa}\right)\end{array}$ \\
\hline A & $1.05,0.44$ & $+0.07(+0.16)$ & $+0.05(+\mathbf{0 . 1 1})$ & $+0.38(+0.86)$ \\
\hline $\mathrm{B}$ & $1.76,0.56$ & $-0.44(-0.79)$ & $-0.28(-\mathbf{0 . 5 0})$ & $+0.95(+1.70)$ \\
\hline $\mathrm{C}$ & $2.28,0.67$ & $-0.15(-\mathbf{0 . 2 2})$ & $-0.18(-0.27)$ & $-0.78(-1.16)$ \\
\hline $\mathrm{D}$ & $1.50,0.60$ & $+0.10(+0.17)$ & $+0.19(+0.32)$ & $-0.07(-\mathbf{0 . 1 2})$ \\
\hline $\mathrm{E}$ & $1.69,0.54$ & $+0.00(-\mathbf{0 . 0 0})$ & $-0.01(-0.02)$ & $-0.26(-0.48)$ \\
\hline $\mathrm{F}$ & $1.51,0.36$ & $-0.05(-0.14)$ & $-0.10(-0.28)$ & $-0.08(-\mathbf{0 . 0 4})$ \\
\hline G & $1.85,0.56$ & $-0.08(-0.14)$ & $+0.02(+\mathbf{0 . 0 4})$ & $-0.02(-0.26)$ \\
\hline $\mathrm{H}$ & $1.33,0.54$ & $+0.12(+\mathbf{0 . 2 2})$ & $+0.14(+0.26)$ & $-0.23(-0.43)$ \\
\hline I & $1.26,0.44$ & $+0.18(+0.41)$ & $+0.12(+0.27)$ & $-0.09(-\mathbf{0 . 2 0})$ \\
\hline $\mathrm{J}$ & $1.64,0.47$ & $+0.06(+0.13)$ & $-0.04(-\mathbf{0 . 0 9})$ & $-0.53(-1.13)$ \\
\hline $\mathrm{K}$ & $1.27,0.57$ & $+0.09(+0.16)$ & $-0.01(-\mathbf{0 . 0 2})$ & $+0.16(+0.28)$ \\
\hline $\mathrm{L}$ & $1.48,0.67$ & $-0.13(-0.19)$ & $-0.12(-\mathbf{0 . 1 8})$ & $+0.35(+0.52)$ \\
\hline M & $1.33,0.33$ & $+0.11(+\mathbf{0 . 3 3})$ & $+0.17(+0.52)$ & $+0.34(+1.03)$ \\
\hline $\mathrm{N}$ & $1.22,0.58$ & $-0.08(-0.14)$ & $-0.09(-0.16)$ & $+0.07(+\mathbf{0 . 1 2})$ \\
\hline $\mathrm{O}$ & $1.11,0.36$ & $-0.01(-\mathbf{0 . 0 3})$ & $-0.02(-0.06)$ & $+0.32(+0.89)$ \\
\hline \multirow[t]{2}{*}{$\mathrm{P}$} & $1.15,0.41$ & $+0.05(+\mathbf{0 . 1 2})$ & $+0.07(+0.17)$ & $+0.68(+1.66)$ \\
\hline & Average absolute & $0.11(0.21)$ & $0.10(\mathbf{0 . 2 0})$ & $0.33(0.68)$ \\
\hline
\end{tabular}

Best results for each image are shown in bold. 
Table 3. Distance from $\hat{a}$; as percentage of true composite cell area $\left(\varepsilon_{a}=(A-\hat{A}) / \hat{A}\right)$ and number of standard deviations $\left(\zeta_{a}=(A-\hat{A}) / \sigma_{\hat{A}}\right)$ for test images.

\begin{tabular}{|c|c|c|c|c|}
\hline Image & $\hat{A}, \sigma_{\hat{A}}$ & $\begin{array}{c}\text { LSEF } \\
\varepsilon A\left(\zeta_{A}\right)\end{array}$ & $\begin{array}{c}\mathrm{AM} \\
\varepsilon A\left(\zeta_{A}\right)\end{array}$ & $\begin{array}{c}\text { CAS } \\
\varepsilon A\left(\zeta_{A}\right)\end{array}$ \\
\hline $\mathrm{a}$ & 2744,1267 & $-78(-1.69)$ & $-41(-0.88)$ & $-7(-\mathbf{0 . 1 4})$ \\
\hline b & 2027,708 & $-43(-1.23)$ & $+1(+\mathbf{0 . 0 2})$ & $+31(+0.88)$ \\
\hline $\mathrm{c}$ & 479,200 & $-43(-1.03)$ & $+16(+\mathbf{0 . 3 8})$ & $+266(+6.36)$ \\
\hline $\mathrm{d}$ & 619,222 & $-71(-1.98)$ & $-28(-\mathbf{0 . 7 9})$ & $+166(+4.63)$ \\
\hline $\mathrm{e}$ & 504,224 & $-44(-0.98)$ & $+32(+\mathbf{0 . 7 2})$ & $+119(+2.69)$ \\
\hline $\mathrm{f}$ & 1010,395 & $+46(-1.17)$ & $+26(+\mathbf{0 . 6 6})$ & $+267(+6.84)$ \\
\hline $\mathrm{g}$ & 658,264 & $-53(-1.33)$ & $-17(-\mathbf{0 . 4 3})$ & $-19(-0.47)$ \\
\hline $\mathrm{h}$ & 297,144 & $-54(-1.11)$ & $+5(+\mathbf{0 . 1 1})$ & $+27(+0.56)$ \\
\hline $\mathrm{i}$ & 472,259 & $-61(-1.12)$ & $-11(-\mathbf{0 . 2 0})$ & $+122(+2.23)$ \\
\hline $\mathrm{j}$ & 476,189 & $-62(-1.57)$ & $-17(-\mathbf{0 . 4 2})$ & $+67(+1.69)$ \\
\hline $\mathrm{k}$ & 412,204 & $-54(-1.09)$ & $+15(+\mathbf{0 . 3 1})$ & $+58(+1.18)$ \\
\hline 1 & 373,158 & $-59(-1.39)$ & $-10(-\mathbf{0 . 2 3})$ & $+70(+1.66)$ \\
\hline $\mathrm{m}$ & 203,79 & $-45(-1.16)$ & $+26(+\mathbf{0 . 6 6})$ & $+69(+1.78)$ \\
\hline $\mathrm{n}$ & 507,276 & $-60(-1.10)$ & $+2(+\mathbf{0 . 0 4})$ & $+107(+1.96)$ \\
\hline o & 208,82 & $-37(-\mathbf{0 . 9 3})$ & $+37(+0.94)$ & $+619(+15.70)$ \\
\hline $\mathrm{p}$ & 211,74 & $-48(-1.36)$ & $+5(+\mathbf{0 . 1 5})$ & $+478(+13.62)$ \\
\hline & Average absolute & 54 (1.27) & $18(\mathbf{0 . 4 3})$ & $156(3.90)$ \\
\hline
\end{tabular}

Best results for each image are shown in bold.

error. The benefit of the latter is that it takes into account the variability of the cells in the image. Discrepancies in $\alpha$ are reported in degrees as $\varepsilon_{\alpha}=\alpha-\hat{\alpha}$ and in normalized form as $\zeta_{\alpha}=\varepsilon_{\alpha} / \sigma_{\hat{\alpha}}$ in table 1 . Discrepancies in $\kappa$ and $A$ are reported in a similar form in tables 2 and 3 .

All methods estimated $\hat{\alpha}$ with similar accuracy and achieved the performance objective of $\left|\zeta_{\alpha}\right|<1$ for all 16 test images. The AM method was the most accurate, with the lowest average $\left|\zeta_{\alpha}\right|(0.19)$ and the lowest maximum $\left|\zeta_{\alpha}\right|(0.53$ for the image in figure 3(n)). All methods had some difficulty calculating $\alpha$ for image: (a) because the fabric is nearly isotropic $(\hat{\kappa}=1.1)$. In contrast, cell orientations in images (c) and (g) are easily detected because in both cases $\hat{\kappa}=1.8$. All methods also had some difficulty with images $(\mathrm{k})$ and $(\mathrm{n})$ because of the lack of a consistent cell orientation, as evidenced by the large value of $\sigma_{\hat{\alpha}}$.

In terms of estimating $\hat{\kappa}$, the AM and LSEF methods were the most accurate. The AM method had the lowest average $\left|\zeta_{\kappa}\right|(0.20)$ and the lowest maximum $\left|\zeta_{\kappa}\right|$ (0.52 for image $\left.(\mathrm{m})\right)$, while the performance of the closely-related LSEF method was nearly as good. The CAS method produced some large errors in estimating $\hat{\kappa}$ because the $|F|$ images often did not have well-defined peaks in the major and minor axes directions.

For calculating $\hat{A}$, the AM method was the most accurate. It achieved the performance objective and had an average $\left|\zeta_{A}\right|$ of 0.43 when equation (8) was used to reduce the correlation between image size and calculated $A$ values. For images with $10-22$ c.p.i., $\left|\zeta_{\kappa}\right|$ was almost always $<0.5$.

Calculations of $\hat{A}$ using the LSEF and CAS methods were not as accurate as those found using the AM approach. The LSEF method consistently underestimates $\hat{A}$ due to its squared distance measure. This causes highfrequency energy to be weighted more strongly causing the fitted ellipse to be larger than it otherwise would be. The CAS on average overestimates $\hat{A}$ due to mistakenly identifying peaks in the $|F|$ image that are caused by lowfrequency noise.

For typical 350 by 350 pixel images, the average computation times for each method (including all preprocessing steps) are less than 1 second on a Pentium 4 running at $2.4 \mathrm{GHz}$.

\section{Applications}

Figure 8(a) shows the blastopore and surrounding epidermis of a stage 12.5 axolotl embryo. The blastopore is a circular gateway from the outside to the inside of the embryo and its location is identifiable by the nodule of cells known as the yolk plug that fills it. At this stage of development a process called gastrulation is occurring and the epidermis flows through the blastopore into the interior of the embryo. The forces that drive this crucial developmental event are of great interest (Belousov and Luchinskaia 1995), and information about cellular fabric would provide important clues about the forces at work.

A 250 by 250 pixel region just lateral to the blastopore is shown in outline in figure 8(a). When the AM algorithm was used to determine the fabric of this region in three different embryos $(n=3)$, it showed that $\kappa$ was $1.65 \pm 0.20$, where the term following the \pm symbol indicates the standard deviation of the embryo-to-embryo variations. The average cell size was $172 \pm 30$ pixels and the angle of elongation $87 \pm 5^{\circ}$. This means that the cells are elongated in approximately the vertical direction of the image and that they are 1.65 times as long as they are wide.

Based on this fabric information, it is possible to show (Brodland et al. 2006) that the cephalo-caudal (verticallyoriented) in-plane tensions acting in this region of the 
(a)

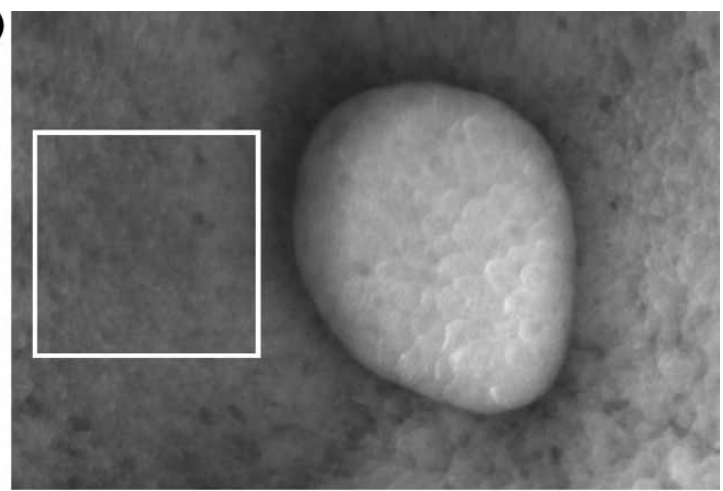

(b)

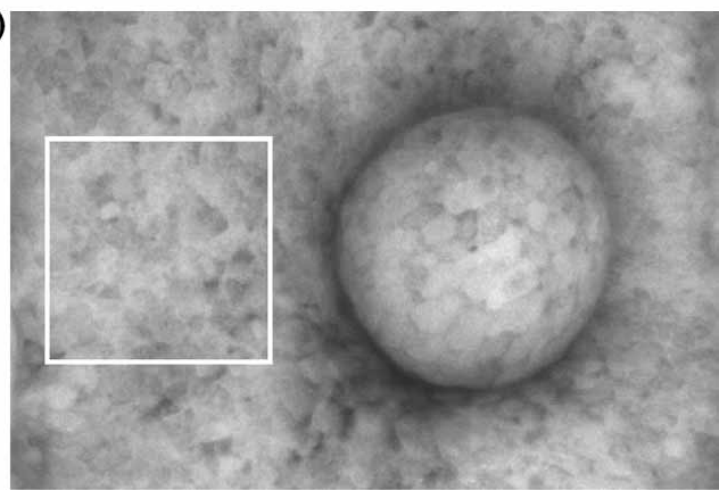

Figure 8. Images showing tissue from the blastopore regions of two stage 12.5 axolotl embryos. In both cases, the midsagittal plane of the embryo bisects the blastopore vertically and the cephalic end of the embryo is oriented upwards. (a) tissue from an intact embryo; (b) tissue from an explant.

embryo are 2.1 times larger than those in the medio-lateral (horizontal) direction. This suggests that the motion of the epidermis toward the blastopore is not driven by radial tension generated at the edge of the blastopore (Carlson 1981), but is instead driven by circumferential forces (Belousov and Luchinskaia 1995). If the blastopore and its surrounding tissue is excised from the embryo (figure 8(b)), the stress in the tissue is relieved and the fabric becomes sensibly isotropic, with $\kappa=1.05 \pm 0.05$ $(n=3)$, supporting this hypothesis.

To investigate the performance of the algorithms further, they were used to monitor fabric changes in an excised epithelium specimen (not shown) that was stretched using a novel test instrument (Wiebe and Brodland, 2005). Stretching the tissue provided a finelygraduated sequence of incremental cell deformations from which the sensitivity of the algorithms could be ascertained. The AM method was again the most reliable (Iles 2005), and it showed that the primary axis of the representative composite cell rotated from approximately $20^{\circ}$ off the stretch axis to within $5^{\circ}$ of it as the cells elongated to a $\kappa$ of 1.6. The cell area increased by a factor of nearly two during that time (Iles 2005). These findings are consistent with findings from computer simulations (Chen and Brodland 2000, Brodland and Wiebe 2004, Brodland and Veldhuis 2003) and they suggest that the $\mathrm{AM}$ approach is able to detect fabric aspect ratio changes of the order of 0.10 and angle changes of $10^{\circ}$.

\section{Discussion and conclusions}

Three computational algorithms with the power to calculate cellular fabric in epithelia were identified. All three of the methods determined $\alpha$ and $\kappa$ to within one standard error of their best estimates and the AM method was also able to reach the cell size target. Although the AM method provides the best results in virtually all cases considered here, the LSEF and CAS approaches are valuable because their relative performance may be better in other situations and because they provide comparative values for evaluation of the AM method. GF did not work well when applied to real tissues.

The AM method is able to extract fabric information even in cases (figure 8) where cell boundaries are so poorly defined that one could not hope to make a visual assessment of the cellular fabric. Strong correlations between tests on corresponding tissues in three different embryos show that measurement uncertainty and embryoto-embryo fabric variations are both relatively small.

In general, an indication of the expected measurement error can be obtained by comparing findings from windows with different sizes and locations in the region of interest. This approach is also useful for revealing regions where substantial fabric gradients are present. If staining, shadowing or other techniques are used to emphasize cell boundaries, care must be taken to ensure that the visible boundaries are representative of all cell boundaries in the tissue.

In practice, the $\mathrm{AM}$ method has been found to work well when certain, potentially conflicting conditions are met. The long axis of the cells in the analysis region should be at least 10 pixels long. Cell boundaries, however, should be no more than one pixel in width, an objective which can be achieved using image binning. In addition, the square analysis region should be $10-20$ cells in length and width. If only aspect ratio and orientation parameters are of interest, the conditions can be relaxed considerably.

Ideally, the lens axis should be kept within $10^{\circ}$ of normal to the tissue being imaged so that foreshortening effects can be neglected. Alternatively, foreshortening effects can be removed by suitably warping the captured images before the fabric extraction algorithm is applied or by mathematically correcting fabric parameters calculated from a foreshortened image.

The algorithms presented here are general in nature, and they could be applied to the geometric characterization of other two-dimensional systems, including cultured cell monolayers, engineered tissue constructs, granular materials, foams and networks.

The present study strengthens our understanding of the relationship between tissue fabric and mechanics. While previous studies showed that fabric is an indicator of the strain history of a tissue and of the stresses at work in it, the present study shows how fabric can be used to reach important new conclusions about how morphogenetic movements are driven. 


\section{References}

J.P. Trinkaus, Cells into Organs, the Forces that Shape the Embryo, 2nd ed., Englewood Cliffs, NJ: Prentice-Hall, 1984.

S.F. Gilbert, Developmental Biology, Sunderland, MA: Sinauer Associates, Inc., 2003.

A.G. Jacobson and R. Gordon, "Changes in the shape of developing vertebrate nervous system analyzed experimentally, mathematically and by computer simulation", J. Exp. Zool., 197, pp. 191-246, 1976.

R.E. Keller, "Time-lapse cinemicrographic analysis of superficial cell behavior during and prior to gastrulation", J. Morphol., 157, pp. $223-248,1978$.

C.O. Jacobson, "Cell migration in the neural plate and the process of neurulation in the axolotl larva", Zool. Bidrag. (Uppsala), 35, pp. $433-449,1962$.

H.H. Chen and G.W. Brodland, "Cell-level finite element studies of viscous cells in planar aggregates", ASME J. Biomech. Eng., 122, pp. 394-401, 2000.

G.W. Brodland and J.H. Veldhuis, "Computer simulations of mitosis and interdependencies between mitosis orientation, cell shape and epithelia reshaping", J. Biomech., 32, pp. 673-681, 2002.

G.W. Brodland, J.H. Veldhuis and D.I.-L. Chen, "How cell shape affects the stresses in a cell sheet", ASME IMECE, Orlando FL, 5-10 November 2000, pp. 79-80.

G.W. Brodland and C.J. Wiebe, "Mechanical effects of cell anisotropy on epithelia", Comput. Methods Biomech. Biomed. Eng., 7(2), pp. 91-99, 2004.

G.W. Brodland, D.I.-L. Chen and J.H. Veldhuis, "A cell-based constitutive model for embryonic epithelia and other planar aggregates of biological cells", Int. J. Plast., 33(6), pp. 821-828, 2006.

P. Ranefall, L. Egevad, B. Nordin and E. Bengtsson, "A new method for segmentation of colour images applied to immunohistochemically stained cell nuclei”, Anal. Cell. Pathol., 15(3), pp. 145-156, 1997.

C. Wählby, J. Lindblad, M. Vondrus, E. Bengtsson and L. Bjrkesten, "Algorithms for cytoplasm segmentation of fluorescence labelled cells", Anal. Cell. Pathol., 24, pp. 101-111, 2002.

D.P. Mukherjee, N. Ray and S.T. Acton, "Level set analysis for leukocyte detection and tracking", IEEE Trans. Image Process., 13(4), pp. 562-572, 2004.

F. Ying-Lun, J.C.K. Chan and R.T. Chin, "Automated analysis of nervecell images using active contour models", IEEE Trans. Med. Imaging, 15(3), pp. 353-368, 1996

W. Lin, J. Xiao and E. Micheli-Tzanakou, "A computational intelligence system for cell classification”, IEEE Int. Conf. Inform. Technol. Appl. Biomed., 16-17 May, pp. 105-109, 1998.
T. Matsuyama, S.-I. Miura and M. Nagao, "Structural analysis of natural textures by fourier transformation", Сотри Vision Graphics Image Process., pp. 347-362, 1983.

H.-B. Kim and R.-H. Park, "Extracting spatial arrangement of structural textures using projection information", Pattern Recognit., pp. $237-245,1992$

V.V. Starovoitov, S.-Y. Jeong and R.-H. Park, "Texture periodicity detection: Features, properties and comparisons", IEEE Trans. Sys. Man Cybern.-Part A: Sys. Humans, 28(6), pp. 839-849, 1998.

J.H. Veldhuis, G.W. Brodland, C.J. Wiebe and G.J. Bootsma, "Multiview robotic microscope reveals the in-plane kinematics of amphibian neurulation", Ann. Biomed. Eng., in press.

C. Wiebe and G.W. Brodland, "Tensile properties of embryonic epithelia measured using a novel instrument", J. Biomech., in press.

W.G. Brodland and C.J. Wiebe, "Mechanical effects of cell anisotropy on epithelia", Comput. methods Biomech. Biomed. Eng, 7(2), pp. 91-99, 2004.

K.R. Castleman, Digital Image Processing, Englewood Cliffs, NJ: Prentice Hall, 1996.

A. Fitzgibbon, M. Pilu and R.B. Fisher, "Direct least square fitting of ellipses", Pattern Anal. Mach. Intell., 21(5), pp. 476-480, 1999.

J. Bigün, G.H. Granlund and J. Wiklund, "Multidimensional orientation estimation with applications to texture analysis and optical flow", IEEE Trans. Pattern Anal. Mach. Intell., 13(8), pp. 775-790, 1991.

G.W. Brodland and J. Veldhuis, "A computer model for reshaping of cells in epithelia due to in-plane deformation and annealing", Comput. Methods Biomech. Biomed. Eng., 6(2), pp. 89-98, 2003.

R.C. Hibbeler, Mechanics of Materials, 6th ed., New Jersey, NJ: Prentice-Hall, 2005.

A.C. Bovik, M. Clark and W.S. Geisler, "Multichannel texture analysis using localized spatial features", IEEE Trans. Pattern Anal. Mach. Intell., 12(1), pp. 55-73, 1990.

D.A. Clausi and M.E. Jernigan, "Designing Gabor filters for optimal texture separability", Pattern Recognition, 33, pp. 1835-1849, 2000.

S.M. Puddister, "Estimating bulk geometrical properties of cellular structures", Masters thesis, University of Waterloo, 2003.

L.C. Belousov and N.N. Luchinskaia, "Mechanically dependent heteropias of the axial rudiments in clawed toad embryos", Ontogenez, 26(3), pp. 213-222, 1995.

B.M. Carlson, Patten's Foundations of Embryology, 4th ed., New York, NY: McGraw-Hill, 1981.

P.J.W. Iles, "Average cell orientation, eccentricity and size estimated from tissue images", Masters thesis, University of Waterloo, 2005. 